\title{
Effect of acoustic velocity on the primary atomization of a hollow cone spray in a swirling flow field
}

\author{
Aravind I B ${ }^{\star 1}$, Rohit Ranjan Bhattacharjee ${ }^{1}$, Satyanarayanan R Chakravarthy ${ }^{1}$ \\ ${ }^{1}$ National Centre for Combustion Research and Development (NCCRD) and, Department of \\ Aerospace Engineering, Indian Institute of Technology Madras, Chennai, India \\ *Corresponding author email: aravindib1992@gmail.com
}

\begin{abstract}
This paper reports an experimental investigation on the effect of imposed acoustic velocity fluctuations on the primary atomization of a hollow cone liquid sheet in the presence of strong air swirl. The atomization dynamics is elucidated by positioning the spray at an acoustic velocity node, antinode, and a mixed point in the standing wave field generated due to the imposed axial acoustic excitation. High speed shadowgraphy images acquired in-sync with dynamic pressure measurements are processed to extract breakup length, spatial growth rates etc. A novel method to obtain the breakup length of a hollow cone spray from the position of maximum wave amplitude is presented. The phase difference between the left and right half-angle fluctuations shows that the flapping motion of the spray is predominantly observed at the mixed point for different air to liquid ratios.
\end{abstract}

\section{Keywords}

Hollow cone spray, air swirl, high speed shadowgraphy, acoustic excitation.

\section{Introduction}

Atomization of a liquid sheet is a critical process particularly in its application in liquid-fuelled propulsion systems. In these combustors, the liquid fuel is oftentimes injected as a hollow cone spray using pressure-swirl simplex atomizers [1]. However, under certain operating conditions, liquid fuelled combustors are susceptible to combustion instability [2]. These are self-excited, large amplitude oscillations in pressure and heat release caused by resonant coupling between combustion and acoustics. These are further complicated by droplet formation, motion and evaporation of the liquid fuel, which can couple with the thermoacoustic oscillations creating time varying fuel delivery to the combustion zone.

Acoustic oscillations affect the spray by forming large-scale vortical structures which entrains droplets, [3, 4] alters velocity field [4] and can increase evaporation rates, [4] thereby decreasing droplet size [5, 6], resulting in finer atomization [7] and enhancing combustion process [8]. These oscillations improve spray development and patternation by changing liquid disintegration characteristics [9, 10]. Studies performed on flat liquid sheets showed that acoustic excitation results in structurally different liquid disintegration characteristics as compared to unexcited flows [10]. In the presence of acoustic oscillations, the interfacial wave development on the liquid sheet reduces surface tension dominated instability [10]. These oscillations augment the growth of sinuous waves responsible for sheet breakup [11], resulting in flag-like flapping $[12,13]$ and synchronizing droplet shedding and axial velocity fluctuations with an excitation frequency $[13,14]$. The liquid sheet breakup processes are enhanced [10], thereby shortening breakup length [6, 9]. Experiments on head-on impinging jets show that the sheet responds only above a certain minimum sound pressure level and this minimum sound pressure level increases with an increase in the excitation frequency [6]. The response is significant at lower frequencies and increases with sound intensity [15]. The instantaneous location of droplets in acoustic field determines the extent to which 
evaporation processes are enhanced [14], with effects being stronger at the acoustic velocity anti-node and minimal at acoustic velocity node $[4,8]$.

In view of this, the effect of an acoustic field on the liquid sheet breakup needs attention to provide important insights into the behaviour of sheet instabilities triggered by perturbations in the pressure and velocity field. Although earlier studies have suggested that acoustic excitation affects droplet formation in a spray, not much has been done to understand the effect of acoustic excitation on the dynamics of primary breakup of sprays. Several investigations have been performed on the effect of acoustic perturbations on flat liquid sheet [6, 11-13, 15], air-assisted flat liquid sheet [10], conical liquid sheet [9, 15], air assisted conical spray [3, 4, 7, 8, 14], swirling air assisted conical spray [5]. However, there is minimal literature about primary atomization of hollow cone sprays in the presence of swirling flows and an imposed acoustic field and the spray characteristics within an acoustic cycle.

In this paper, an experimental investigation is performed, on a non-reactive flow, to understand the effect of acoustic velocity fluctuations on the primary atomization dynamics of a hollow cone spray from a pressure swirl nozzle in a strong air swirl using high-speed back-light imaging, The effect of imposing an acoustic velocity on the dynamics of primary atomization is investigated by comparing the spray positioned at three different locations in standing wave field - an acoustic velocity node, acoustic velocity antinode, and a mixed point with non-zero acoustic pressure and acoustic velocity (hereafter called as pressure-velocity). For a cold flow problem, as studied here, self-excitation is not possible therefore necessitating an external acoustic forcing in one direction. However, acoustic excitation in one direction (axial or transverse) represents only a canonical formulation of the combustion instability problem seen in real gas turbine engines. In the combustion chamber, all three acoustic modes may be present i.e., axial, transverse and radial [2] in addition to acoustic modulation that may be present within the nozzle itself [9]. Considering the strong acousticfluid flow coupling that is present [2], the simplification of considering only one mode at a time can potentially obfuscate the full physics at play. Nevertheless, since the objective of this study is to isolate and elucidate the impact of axial acoustic excitation has on the spray dynamics, we shall adopt this canonical approach. In the next section, the experimental setup and methods are described, followed by the presentation of results and their discussion. The paper closes with a summary of the main conclusions.

\section{Material and Methods}

The experimental setup used for this study comprises a plenum chamber, two loudspeakers (Dainty DSW 12R, $200 \mathrm{~W}$ RMS). The test section, where spray - swirling field is enclosed, is made square $\left(140 \mathrm{~mm}{ }^{*} 140 \mathrm{~mm}\right.$ ) to provide for flat windows. This prevents any distortion in the images obtained due to using curved windows. The air flow rate is metered using mass flow controllers from Alicat Scientific Inc (Serial No.60207). In order to study the effect of acoustic velocity on the primary atomization of the hollow cone spray, the duct has a modular design allowing the test section to be placed at different locations in the standing wave field. The three different configurations are shown in Figure 1.

The loud speakers are attached downstream of the test section using extension ducts to provide longitudinal excitation from the downstream end. A choke plate is used to satisfy the closed acoustic boundary conditions. Flow straightener is used to ensure the flow downstream of the choke plate is uniform. The loud speakers are excited with a sinusoidal wave of specific frequency and amplitude produced by a signal generator (Tektronix AFG 2021) and amplified by an amplifier (Ahuja UBA-500M). The frequency of the sinusoidal signal can be altered by varying the waveform generated in the signal generator. Piezo- 
electric pressure transducers from PCB Piezotronics Inc. (model 103B02), with sensitivity of $217.5 \mathrm{mV} / \mathrm{kPa}$, are used for dynamic pressure measurements. Constant voltage is supplied to the speakers and the response of the duct is measured using piezoelectric transducers.

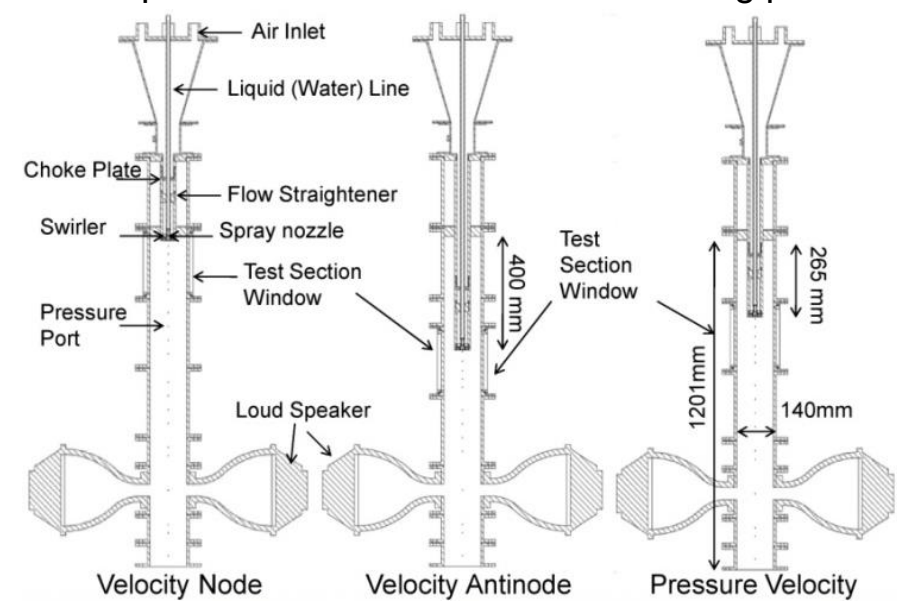

Figure 1. Schematic of experimental setup with spray nozzle positioned at different locations

The liquid (water) is pressurized using nitrogen, and ejected through a commercial hollow cone pressure swirl nozzle (BK Series Hollow cone Spray Nozzle) from Spraytech Systems with nozzle orifice diameter, $\mathrm{d}_{\mathrm{o}}$ of $0.7 \mathrm{~mm}$. The liquid Reynolds number, $\mathrm{Re}_{1}$ is calculated as $\operatorname{Re}_{1}=\frac{\rho_{1} U_{1} t}{\mu_{1}}$

where the mean liquid velocity at exit, $\mathrm{U}_{\mathrm{l}}$ is given by [16] $\mathrm{U}_{\mathrm{l}}=\frac{4 \dot{\mathrm{m}}_{1}}{\pi \rho_{\mathrm{l}} \mathrm{d}_{\mathrm{o}}{ }^{2}(1-\mathrm{X})}\left(\frac{1+\mathrm{X}}{1-\mathrm{X}}\right)^{0.5}$ with, $\mathrm{X}=$ $\frac{A_{a}}{A_{o}}=\frac{\left(d_{0}-2 t\right)^{2}}{d_{o}{ }^{2}}$. The liquid film thickness, $t$ is calculated by assuming that the internal flow area occupied by the liquid sheet is $40 \%$ of the total internal orifice area [17]. The liquid mass flow rate $\dot{\mathrm{m}}_{\mathrm{l}}$ is calculated using quadratic polynomial fitting of the flow rate data obtained from the spray nozzle manufacturer. The experiments are carried out at atmospheric ambient pressure for injection pressure differential across nozzle, $\Delta \mathrm{P}$ values of 3 and 4 bars in the present study. The variation of liquid mass flow rate, $\dot{\mathrm{m}}_{1}$ and liquid Reynolds number, $\operatorname{Re}_{1}$ with injection pressure differential across nozzle, $\Delta \mathrm{P}$ is shown in Figure 2 (i).
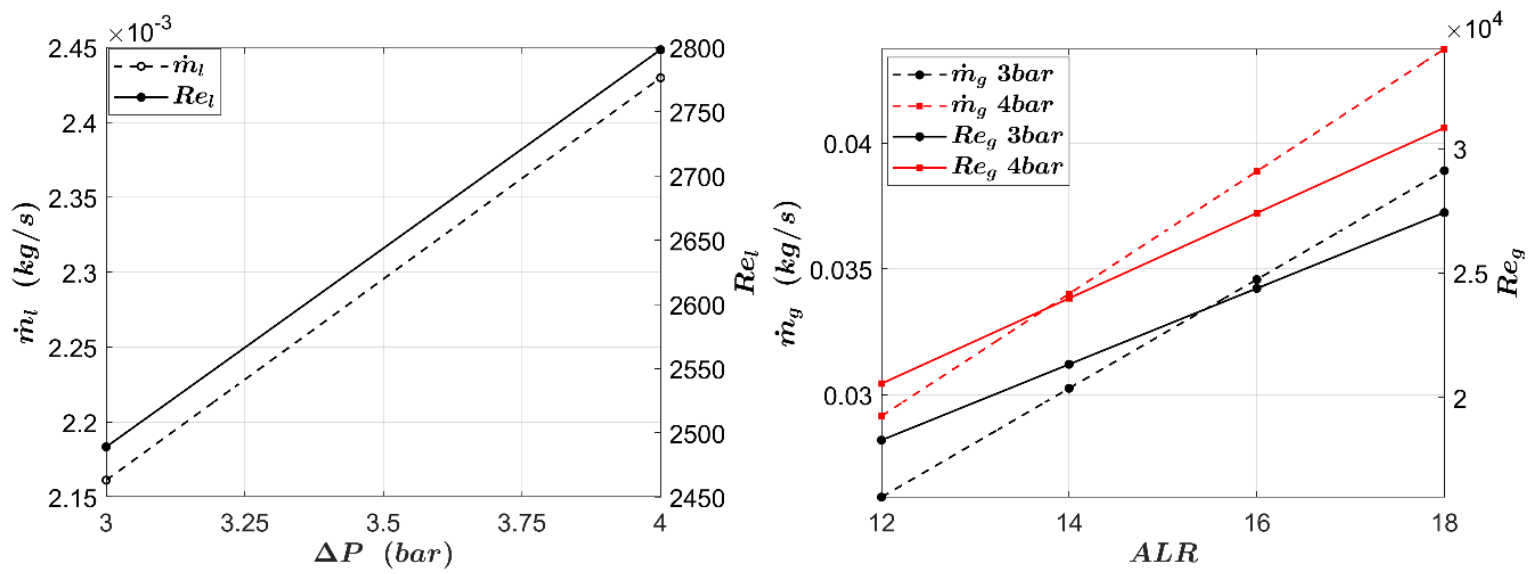

Figure 2 (i). Liquid mass flow rate $\dot{\mathrm{m}}_{1}$ and liquid Reynolds number $\mathrm{Re}_{1}$ vs injection pressure differential across nozzle, $\Delta \mathrm{P}$; (ii) Air mass flow rate $\dot{\mathrm{m}}_{\mathrm{g}}$ and air Reynolds number $\operatorname{Re}_{\mathrm{g}}$ vs ALR for different $\Delta \mathrm{P}$

The swirler used in the present study is an axial flow flat-vane type with geometric swirl number of 0.961 . The simplex nozzle is mounted on the swirler hub such that its centreline axis and the swirler axis are coincident and the nozzle is flush with swirler exit plane. The air Reynolds number is calculated as 
$\operatorname{Re}_{\mathrm{g}}=\frac{\rho_{\mathrm{g}} \mathrm{U}_{\mathrm{g}} \mathrm{D}_{\mathrm{eff}}}{\mu_{\mathrm{g}}}$

with mean air velocity at exit, $U_{g}$ given by, $U_{g}=\frac{\dot{m}_{g}}{\rho_{g} A_{s w}}$. $A_{s w}$ is the frontal area or the effective flow area for the axial flow flat-vane type swirler given by [18],

$A_{\text {sw }}=\left(\frac{\pi}{4}\right)\left(D_{s w}^{2}-D_{\text {hub }}^{2}\right)-0.5 n_{v} t_{v}\left(D_{\text {sw }}-D_{\text {hub }}\right)$

where, $D_{g, e f f}$ is the effective swirler exit diameter for air flow calculated as [17],

$\mathrm{D}_{\mathrm{g}, \mathrm{eff}}=\left(\mathrm{D}_{\mathrm{sw}}-\mathrm{D}_{\text {hub }}\right)-0.5 \mathrm{n}_{\mathrm{v}} \mathrm{t}_{\mathrm{v}}$

The air mass flow rate for the present study is selected so that the air-to-liquid ratio (ALR) is in the range of 12 to 18 . The ALR is defined as the ratio between mass flow rates of air and liquid. The variation of air mass flow rate $\dot{\mathrm{m}}_{\mathrm{g}}$ and air Reynolds number, $\mathrm{Re}_{\mathrm{g}}$ with $\mathrm{ALR}$ for $\triangle \mathrm{P}$ value of 3 and 4 bars is shown in Figure 2 (ii). All test cases were carried out for the three different locations in standing wave field.

\section{Results and Discussion}

\section{Dynamic Pressure Measurement Experiments}

Acoustic tests are performed over a frequency range between $50-400 \mathrm{~Hz}$ in steps of $5 \mathrm{~Hz}$ at a constant amplitude, to estimate the harmonic content of the setup as shown in Figure 3 (i).
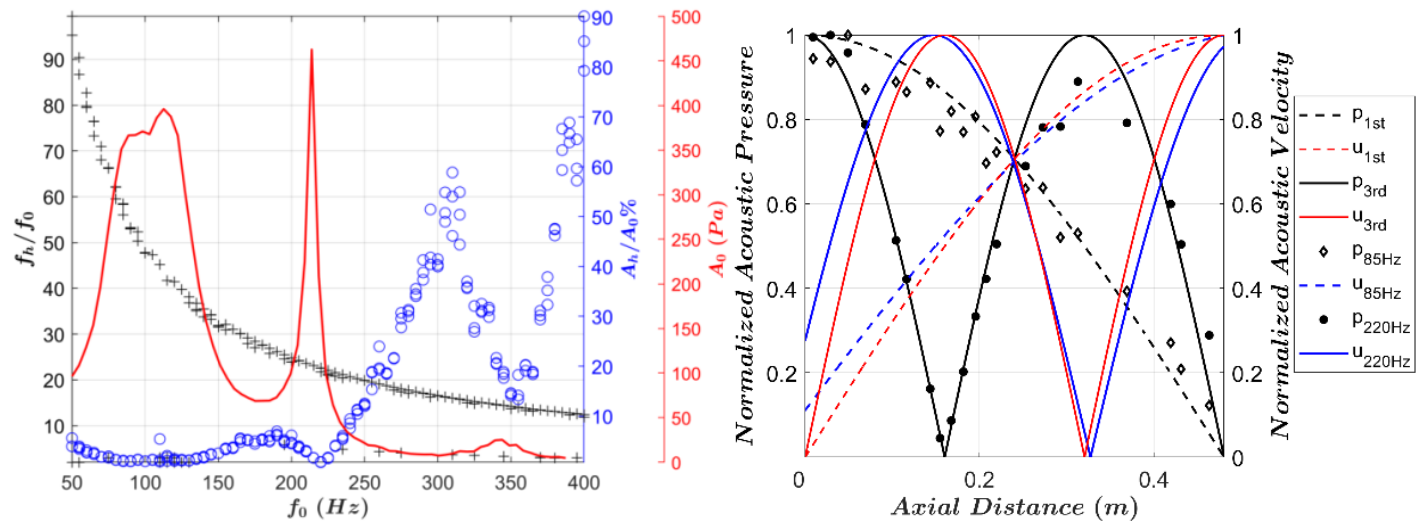

Figure 3. (i) Harmonic content of acoustic excitation; (ii) Modes shapes for fundamental and third harmonic of acoustic pressure and velocity

Here, $f_{0}$ and $A_{0}$ are the forcing frequency and amplitude respectively, and $f_{h}$ and $A_{h}$ are the harmonic frequency and amplitude respectively. The amplitudes of the harmonics are observed to be high for frequencies greater than $250 \mathrm{~Hz}$. Since acoustic excitations at specific frequencies are of interest here, there should be no high amplitude acoustic pressure oscillation for harmonics. The harmonic content is less than $10 \%$ for frequency range between $50 \mathrm{~Hz}$ and $250 \mathrm{~Hz}$ and is selected for the present study. The fundamental frequency calculated for the duct of length $1201 \mathrm{~mm}$ where the standing wave field is established with one end open and another end closed is $72 \mathrm{~Hz}$ and the third harmonic frequency is $216 \mathrm{~Hz}$. The mode shapes of first two harmonics are calculated for this duct length and boundary conditions. The frequency at which the spray is to be excited is selected from the amplitude of pressure oscillations versus forcing frequency as shown in Figure 3 (i). Sharp resonant peak is observed at $220 \mathrm{~Hz}$ and a broad peak around $100 \mathrm{~Hz}$.

Pressure measurements were also taken at different locations in the setup to find the variation of resonant harmonic frequencies along the standing wave field. A frequency of $85 \mathrm{~Hz}$ was found closely matching the 1 st harmonic of the duct and $220 \mathrm{~Hz}$ was found resonating as the 3rd harmonic of the duct. The observed and theoretical mode shapes for acoustic pressure and calculated acoustic velocity of these two harmonics, for this duct 
length and boundary conditions, is shown in Figure 3 (ii). Dynamic pressure measurements using calibrated piezoelectric pressure transducers are performed for all the test cases for the three different locations in standing wave field. Two-microphone technique is used to obtain acoustic velocity $(\mathrm{m} / \mathrm{s})$ at the spray exit location from the dynamic pressure measurements [19]. The imposed acoustic velocity remains constant for the different ALR values and is the highest at velocity antinode and lowest at velocity node and in between the two at pressure velocity.

\section{High-speed imaging experiments}
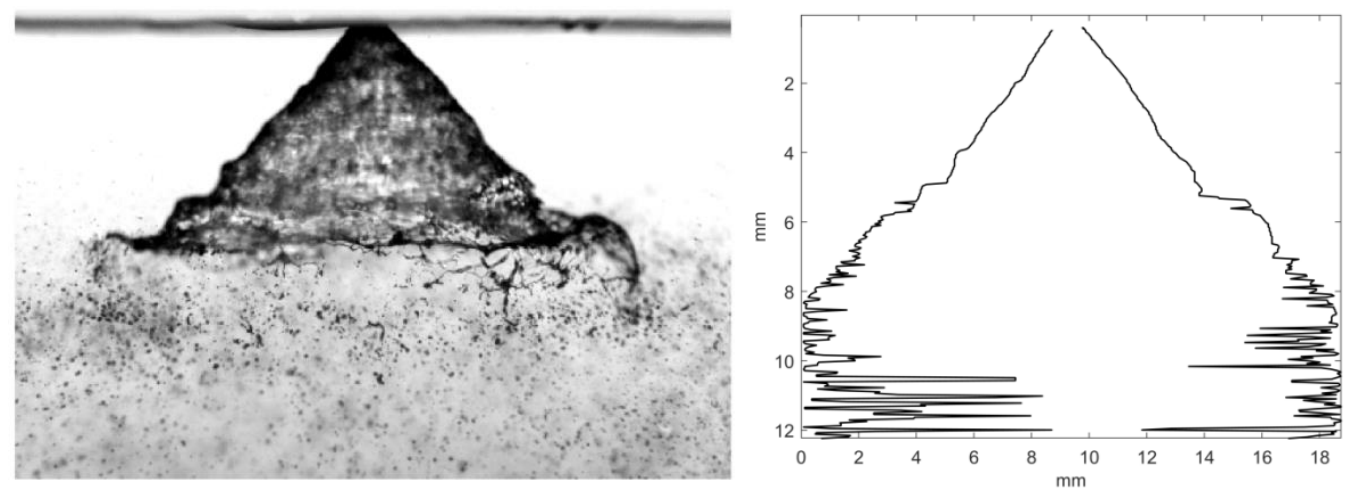

Figure 4 (i). Instantaneous spray image; (ii) Instantaneous spray edge image

High-speed shadowgraph or back-light imaging is carried out for all the test cases using a Phantom high-speed camera at 11000 frames per second with resolution of $1024 \times 512$ pixels ${ }^{2}$ and an exposure of $2.5 \mu \mathrm{s}$. A single LED based strobe light source to illuminate the spray is placed far behind the focal plane of the camera forming a clear white background image. A zoom lens (Sigma AF 70-300 mm F/4-5.6 APO DG Macro) is used to focus at the spray exit allowing a field of view of $47 \mathrm{~mm} \times 24 \mathrm{~mm}$. The pressure transducers are synchronized with the camera to sample at $11 \mathrm{kHz}$. A total of 32000 images are acquired and saved as 8-bit grey scale images. A scale image is taken before the experiments to obtain the magnification factor for converting the distances in pixels to $\mathrm{mm}$. The instantaneous images as shown in Figure 4 (i) are obtained for a statistically steady spray with pressure data acquisition synchronized with imaging. The instantaneous images are pre-processed, using MATLAB, to obtain the spray edge image as shown in Figure 4 (ii). This procedure is repeated for all the 32000 images to obtain spray edges image sequence. Similarly, spray edges image sequences are obtained for all the test cases for the three different locations in standing wave field.

\section{Spray cone angle}

From each spray edge images, the cone angle of the spray is obtained from the slope made by the left and right edges with the spray axis by fitting a straight line to it. From this, the fluctuations of left and right half angles are obtained as a function of time since the sampling rate is known. The mean value of the cone angle, obtained from the mean left and right half angles are shown for all the test cases and all three locations in standing wave field in Figure 5 (i). We find that cone angle increases with ALR, but remains nearly constant for the three duct configurations. Information about dominant fluctuation frequency is obtained through FFT of left and right half angle fluctuation. The left and right half angles oscillate near the excitation frequency of $220 \mathrm{~Hz}$ for lower values of liquid injection pressure and ALR values for the different configurations. However, the frequency deviates from the excitation frequency for higher values of liquid injection pressure and ALR values for the different 
configurations. The phase difference between the fluctuation of the angle made by the left and right edge with the spray axis is obtained from the initial phases of the fluctuation of the left and right half angles and is shown for all excited cases for the three different duct configurations in Figure 5 (ii). Here VN, PV and VA cases are shown at different radial distances only for clarity and has no relevance to the magnitude of the phase difference.
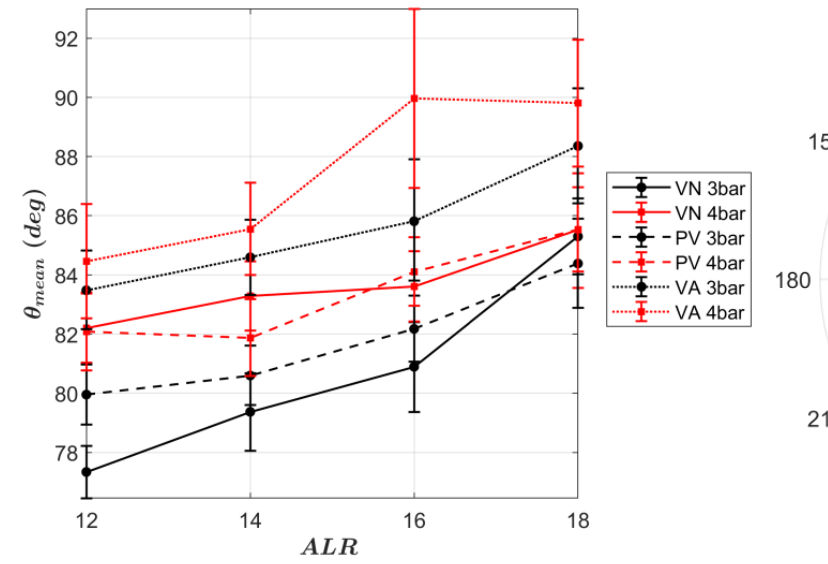

120

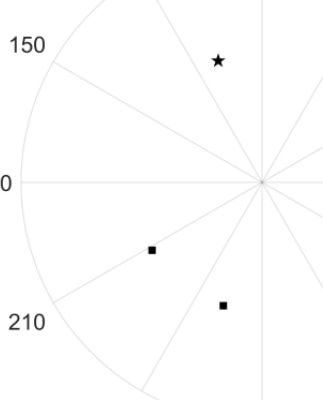

240
60

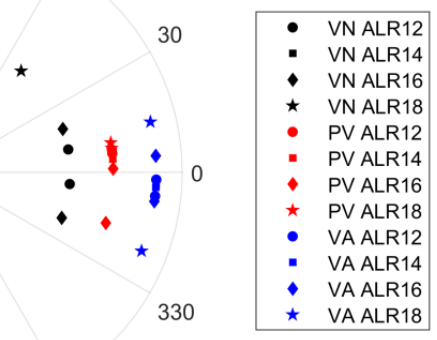

300

Figure 5 (i) Mean Cone Angle vs ALR; (ii) Phase difference between left and right angle for different ALR (VN - Velocity Node, PV-Pressure Velocity, VA - Velocity Antinode)

The phase difference between the fluctuations in the left and right half angles, measured with respect to the spray axis, is used to determine if the spray flaps or not. If both the left and right arm of liquid sheet flaps together, then the left and right half angles increase and decrease simultaneously with zero phase difference. If the phase difference is $180^{\circ}$, then the spray is presumably precessing. The spray flapping motion is observed to be predominant in the acoustic pressure velocity point for different air to liquid ratios.

\section{Spray breakup length}

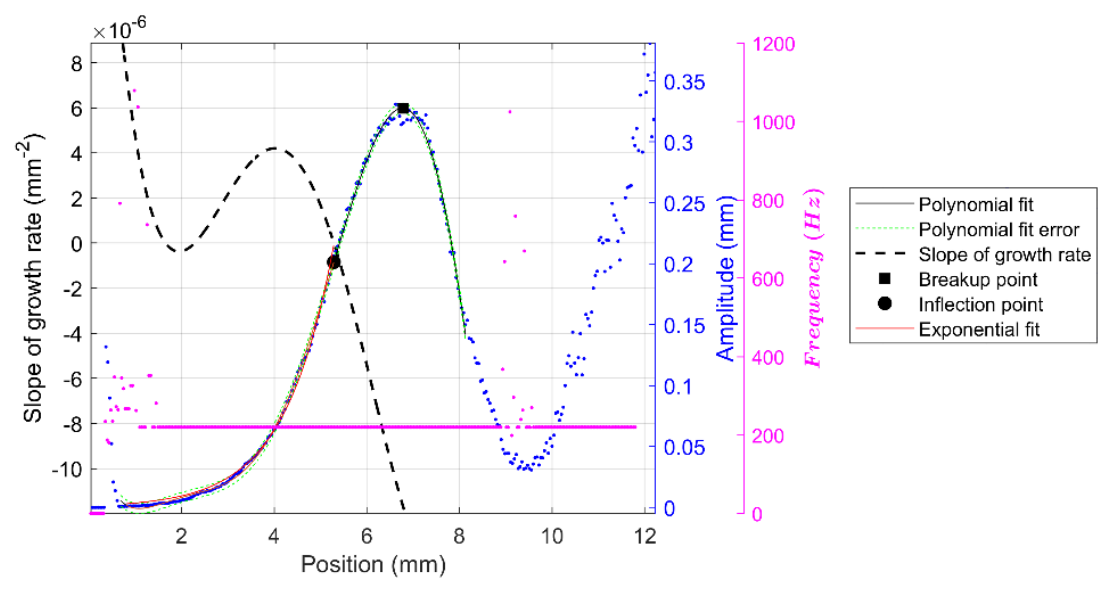

Figure 6. Fluctuation frequency and amplitude vs position

From the spray edges images, the pixel fluctuations in the left and right edge at each downstream location can be obtained as a function of time. On performing an FFT of this time series for the left and right side, the fluctuation frequency and corresponding amplitude in the position of left edge and right edge is obtained. A representative plot of dominant fluctuation frequency and corresponding amplitude along the spray axis for the left edge is shown in Figure 6. From this the position where the amplitude value grows to a maximum, obtained by fitting polynomials of different order, is taken as an estimate of the breakup length. This is because the waves on the liquid sheet grow in amplitude till, they breakup 
from the sheet. The maximum amplitude position remains constant for polynomial orders above 5 for all the cases. The mean of the maximum amplitude position, obtained from fitting polynomials of order 5 to 10 , on each side is taken as the breakup length for that side. The smaller value among the left and right side is taken as the breakup length of the spray and is obtained for all the test cases for the three different locations in standing wave field and is shown in Figure 7 (i).
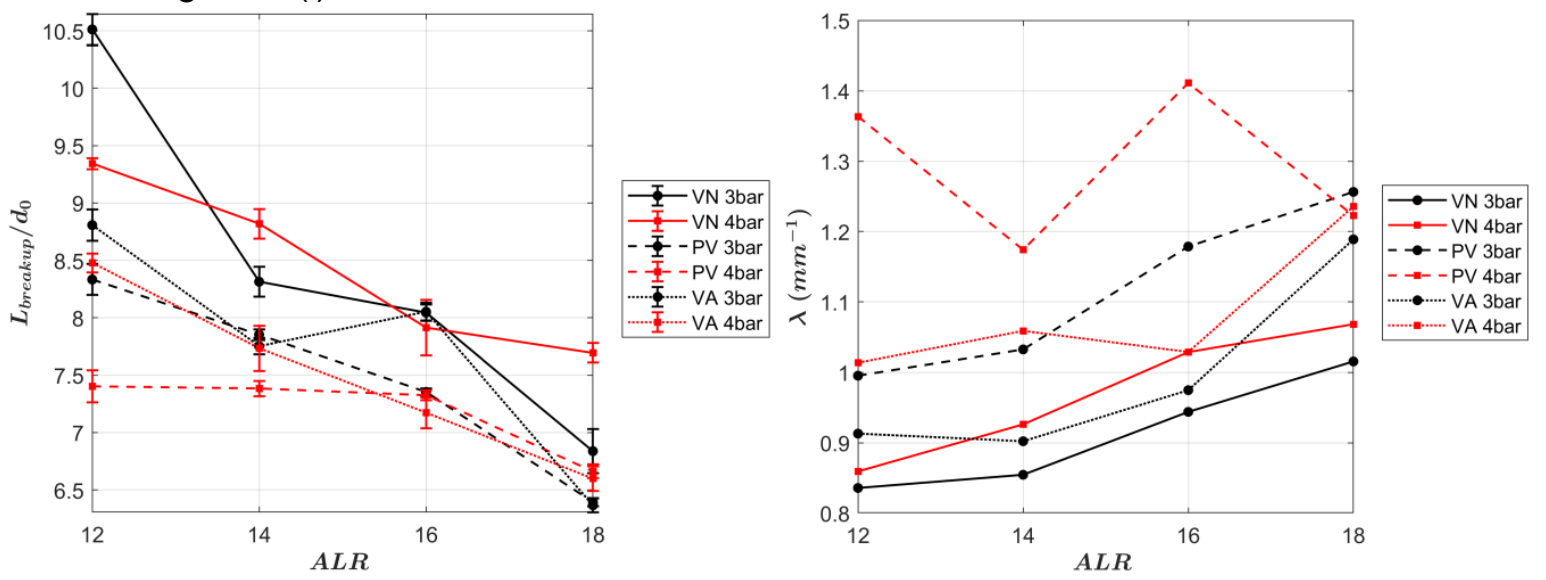

Figure 7 (i) Breakup length vs ALR; (ii) Spatial growth rate of amplitude vs ALR

The breakup length reduces with the ALR and is the lowest for pressure velocity case and highest for velocity node case. This implies that atomization is highly efficient in the presence of both acoustic pressure and acoustic velocity fluctuations and least efficient in the presence of acoustic pressure fluctuations alone.

The polynomial fit that has the position of maximum amplitude closest to the mean value is taken as the best fitting polynomial. Taking the second derivative of the best fitting polynomial with respect to position reveals that there exists an inflection point before the breakup point. In order to find the spatial growth rate of the amplitude, an exponential function of the form $A e^{\lambda x}$ is fitted to the best fitting polynomial till the inflection point. This is performed for the left and right side. The spatial growth rate of amplitude, $\lambda$ for the side with smaller value of breakup length is taken as the spatial growth rate of amplitude for the spray. This is obtained for all the test cases for the three different spray locations and is shown in Figure 7 (ii). The spatial growth rate increases with ALR and is the highest for pressure velocity point and lowest for velocity node point. This implies that waves grow spatially fastest in the presence of both acoustic pressure and acoustic velocity fluctuations and slowest in the presence of acoustic pressure fluctuations alone. The combined effect of fluctuations in acoustic pressure and acoustic velocity respectively causes the liquid mass flow rate to fluctuate by affecting the liquid injection pressures and also directly affecting the shear layer causing it to fluctuate predominantly.

\section{Conclusions}

High speed shadowgraph experiments are performed to study the effect of acoustic velocity fluctuations on the primary atomization of a hollow cone liquid sheet in the presence of strong air swirl when positioned at three different locations in standing wave field generated due to the imposed axial acoustic excitation. The spray flapping motion is observed to be predominant in the mixed point for different ALR. A novel method to obtain the breakup length of a hollow cone spray from the position of maximum wave amplitude is presented. The breakup length is the lowest for the mixed point implying high atomization efficiency in the presence of both acoustic pressure and acoustic velocity fluctuations. The spatial growth 
rate is the highest for the mixed point implying that waves grow spatially fastest in the presence of both acoustic pressure and acoustic velocity fluctuations.

\section{Acknowledgments}

The Science and Engineering Research Board, Department of Science and Technology (DST), Government of India supports the National Centre for Combustion Research and Development (NCCRD).

\section{Nomenclature}

$A \quad$ amplitude of the liquid sheet at the spray nozzle exit [mm]

$A_{o}, A_{a}, A_{s w} \quad$ nozzle orifice, internal air core and swirler effective flow area respectively $\left[m^{2}\right]$

$\mathrm{d}_{\mathrm{o}}, \mathrm{D}_{\mathrm{g}, \mathrm{eff}} \quad$ nozzle orifice, swirler effective exit diameter $[\mathrm{m}]$

$\dot{\mathrm{m}}_{\mathrm{l}}, \dot{\mathrm{m}}_{\mathrm{g}} \quad$ liquid, air mass flow rate respectively $\left[\mathrm{kg} \mathrm{s}^{-1}\right]$

$\mathrm{Re}_{\mathrm{l}}, \mathrm{Re}_{\mathrm{g}} \quad$ liquid, air Reynolds number respectively

$\mathrm{t}, \mathrm{t}_{\mathrm{v}} \quad$ liquid film thickness, swirler vane thickness respectively [m]

$\mathrm{U}_{\mathrm{l}}, \mathrm{U}_{\mathrm{g}} \quad$ mean liquid, air velocity at exit respectively [ $\left.\mathrm{m} \mathrm{s}^{-1}\right]$

$x$

$\rho_{1}, \rho_{\mathrm{g}}$

$\mu_{1}, \mu_{\mathrm{g}}$

$\lambda$

axial downstream distance measured from the spray nozzle exit [mm]

$\Delta \mathrm{P}$

$\mathrm{n}_{\mathrm{v}}$ liquid, air density respectively $\left[\mathrm{kg} \mathrm{m}^{3}\right]$

liquid, air dynamic viscosity respectively [ $\mathrm{N} \mathrm{s} \mathrm{m}^{-2}$ ]

spatial growth rate $\left[\mathrm{mm}^{-1}\right]$

injection pressure differential across nozzle (bars or $10^{5} \mathrm{~Pa}$ )

number of vanes

\section{References}

[1] Saha, Abhishek., Lee, Joshua D., Basu, Saptarshi., Kumar, Ranganathan., 2012, Physics of Fluids, 24, 124103.

[2] Candel, S., Durox, D., Schuller, T., Bourgouin, J. F., Moeck, Jonas P., 2014, Dynamics of Swirling Flames. Annual Review of Fluid Mechanics, 46, pp. 147-173.

[3] Swanson, T. R., Richards, C. D., 1997, Atomization and Sprays, 7, pp. 561-579.

[4] Sujith, R. I., 2005, Experiments in Fluids, 38, pp. 566-587.

[5] Gurubaran, R. Kumara., Sujith, R. I., 2011, International Journal of Spray and Combustion Dynamics, 3, pp. 1-22.

[6] Mulmule, A. S., Tirumkudulu, M. S., Ramamurthi, K., 2010, Physics of Fluids, 22(2), pp. 022-101.

[7] Swamy, K. M., Narayana, K. L., Murty, J. S., 1989, Fuel, 68, pp. 387-390.

[8] Dubey, R. K., Black, D. L., McQuay, M. Q., Carvalho, J. A. Jr., 1997, Combustion and Flame, 110, pp. 25-38.

[9] Chung, I. P., Presser, C., Dressler, J. L., 1998, Atomization \& Sprays, 8(5), pp. 479-502.

[10] Sivadas, V., Heitor, M. N., 2002, Annals of the New York Academy of Sciences, 972, pp. 292-298.

[11] Dighe, S., Gadghil, H., 2019, Journal of Fluid Mechanics, 880, pp. 653-683.

[12] Crapper, G. D., Dombrowski, N., Pyott, G. A. D., 1975, Proceedings of the Royal Society A, 342(1629), pp. 209-224.

[13] Dighe, Sandip., Gadgil, Hrishikesh., 2018, International Journal of Multiphase Flow, 99, pp. 347-362.

[14] McQuay, M. C., Dubey, R. K., 1998, Atomization and Sprays, 8(4), pp. 419-437.

[15] Rhys, N. O., 1999, PhD thesis, The University of Alabama in Huntsville.

[16] Rizk, N., Lefebvre, A., 1985, Journal of Propulsion and Power, 1, pp. 193-199.

[17] Rajamanickam, K., Basu, S., 2017, Journal of Fluid Mechanics, 810, pp. 82-126.

[18] Lefebvre, A. H., Ballal, D. R., 2010, "Gas turbine combustion."

[19] Seybert, A. F., Ross, D. F., 1977, Journal of Acoustical Society of America, 61, pp. $1362-1370$. 